(C)2007 IEEE. Personal use of this material is permitted. However, permission to reprint/republish this material for advertising or promotional purposes or for creating new collective works for resale or redistribution to servers or lists, or to reuse any copyrighted component of this work in other works must be obtained from the IEEE 


\section{Exploring the Conceptual Model of Digital Ecosystem}

\author{
Hai Dong \\ Curtin University of \\ Technology \\ hai.dong@cbs.curtin.edu.au
}

\author{
Farookh Khadeer Hussain \\ Curtin University of \\ Technology \\ hussain.farookh@cbs.curtin. \\ edu.au
}

\author{
Elizabeth Chang \\ Curtin University of \\ Technology \\ elizabeth.chang@cbs.curtin. \\ edu.au
}

\begin{abstract}
Digital Ecosystem, as a neoteric terminology, has emerged along with the appearance of Business Ecosystem which is a form of naturally existing business network of small and medium enterprises. However, few researches have been found in the field of defining digital ecosystem. In this paper, by means of ontology technology as our research methodology, we propose to develop a conceptual model for digital ecosystem. By introducing an innovative ontological notation system, we create the hierarchical framework of digital ecosystem form up to down, based on the related theories form Digital Ecosystem and Business Intelligence Institute.
\end{abstract}

\section{Introduction}

Digital Ecosystem is a neoteric terminology and its appearance is as a result of the natural existence of Business Ecosystem, along with the evolution of business network and information technology. The goal of digital ecosystems is to improve the efficiency of the communication between internal agents and to structuralize the existing Business Ecosystem. Because the researches on digital ecosystem are still in the beginning stage, some basic concepts are in debate. The definition of digital ecosystem is one of them, which have two major opinions. One is defined by Soluta.net from the structural and functional perspective, which sees digital ecosystem as an opensource network environment for business mainly including small and medium enterprises to interact with others in an effective and efficient way [3]. Another one is defined by Digital Ecosystem and Business Intelligence Institute (DEBII) from the perspective of specialization, which sees it as "an open, loosely coupled, domain clustered, demand-driven, self-organizing and agent-based environment which each species is proactive and responsive for its own benefit and profit" [2] [4]. The former definition and related theories focus on the introduction and implementation of the digital ecosystem architecture from pure technical aspect. By constructing the service-oriented environment by means of network technology, BML (Business Modeling Languages), SDL (Service Description Languages), ontology et al., digital ecosystem realizes the function of service creation, service execution and service optimization [3]. The latter definition and based theories concentrate on defining the existing concepts and network architecture and swarm intelligence in digital ecosystem, which tends to conceptualize and structuralize its components. Thus, the former definition is preferable to the actual implementation and commercialization, and the latter definition more inclines to the conceptualization.

To solve the conceptual ambiguity and help researcher better understand what digital ecosystem is, we employ ontology which is seen as an affective tool to assist people share knowledge, to represent the conceptual model of digital ecosystem. Due to the mission of this paper is to conceptualize the components of digital ecosystem, the theory on which our research bases mainly concentrates on the second definition we discussed above.

\section{Notation system for ontology representation}

Before we represent the ontology model for project track and trace, a notation system is introduced in this section. The notation system utilized in the ontological representation is based on Chang et al. [1]'s work, which consists of three basic notations as Table 1. Although in the past we usually used to employ UML to represent ontology model, due to its complex symbols categories, UML cannot efficiently help people better understand the shared knowledge. This notation system simplifies the symbols and its symbols 
are closer to the principle of ontology which is the combination of shared concepts and relationships between concepts [4].

Table 1. Ontology notation system

\begin{tabular}{|c|c|}
\hline $\begin{array}{l}\text { Ontology } \\
\text { Notation }\end{array}$ & Semantics of the Notation \\
\hline & $\begin{array}{l}\text { Double-field Box represents the } \\
\text { Ontological Concepts. }\end{array}$ \\
\hline$--^{\text {relation }}-$ & $\begin{array}{l}\text { A dotted line represents Ontology } \\
\text { Concept Association Relation which } \\
\text { represents a Concept is closely related } \\
\text { to another concept. The relationship } \\
\text { name can be noted above the dotted } \\
\text { line. }\end{array}$ \\
\hline & $\begin{array}{l}\text { Open-arrow line represents } \\
\text { Composition and Aggregation or Part- } \\
\text { of relationship between Upper } \\
\text { Ontology Concept and Lower } \\
\text { Ontology Concept. }\end{array}$ \\
\hline
\end{tabular}

\section{Digital Ecosystem Ontology}

\subsection{Hierarchy of Digital Ecosystem Ontology concepts}

Digital ecosystem consists of two basic parts species and an ecosystem environment. Each species can be viewed as an individual or an organization and has its own role to play. The species work together to take care of their living environment; on the other hand, environment supports species' survival and development. There are three basic types of species biological species, economic species and digital species. Thus, the hierarchy of digital ecosystem concepts is shown in Fig. 1 through the use of notation system.

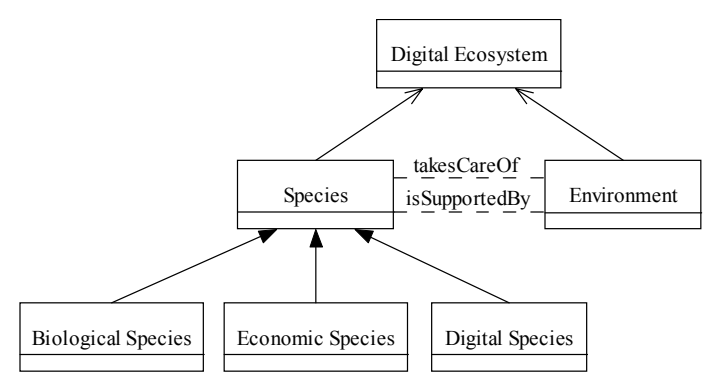

Figure 1. Digital ecosystem concepts hierarchy

\subsection{Species Ontology}

Species is the basic element of digital ecosystem, which can be defined as the conceptualization of Species which interacts with other Species, shares
Commonly Agreed Vocabulary such as Ontology, comes from certain Domain, plays dual Roles including Server who provides Available Services and Client who needs Requested Service, follows Rules of digital ecosystem, is proactive for or is responsive for own Profit or Benefit and carried out Tasks that relates to Profit or Benefit. (Fig. 2)

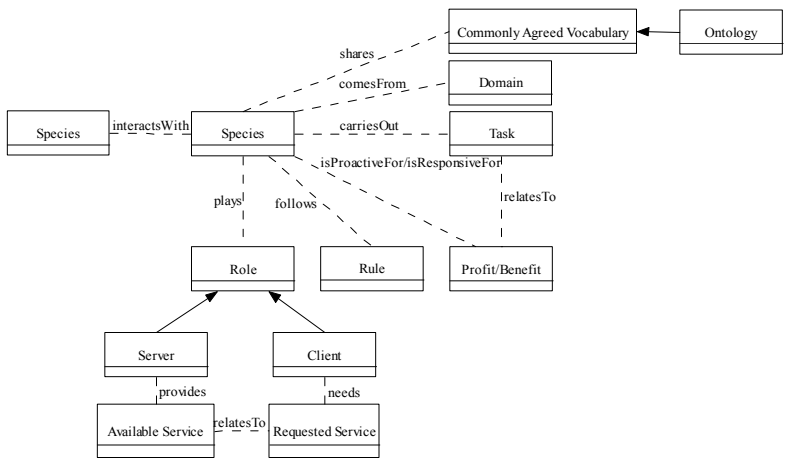

Figure 2. Species Ontology

We present the Species Ontology as the combination of ontology name and a tuple where the elements of the tuple can be complex elements as defined below:

Species [Species, Commonly Agreed Vocabulary, Ontology, Domain, Task, Profit/Benefit, Rule, Role, Server, Client, Available Service and Requested Service] where

'Species' refers to the definitions above.

'Commonly Agreed Vocabulary' refers to the common terms used by species to communicate with each other [4].

'Ontology' is a conceptualization of knowledge in a special domain [5].

'Domain' refers to a field in digital ecosystem where a species has common interest with other species [4].

'Task' refers to a mission that a species is executing, to survive and achieve its goals in the environment of digital ecosystem.

'Profit' or 'Benefit' means a social or economical advantage that a species gains by digital ecosystem [4].

'Rule' refers to a common or special regulation that a species should follow in the environment of digital ecosystem.

'Role' refers to the role of a species in the interaction with other species. There are two major roles in digital ecosystem interactions - server and client. Differed from other network architectures in which each agent has fixed role, each species in digital ecosystem are able to play the dual roles at the same time [4]. 
'Server' is a role of a species that provides a service [4].

'Client' is a role of a service that requests a service [4].

'Available Service' is a service that a supplier provides [4].

'Requested Service' is a service that a requester needs [4].

\subsection{Biological Species Ontology, Economic Species Ontology and Digital Species Ontology}

The three subclasses of species - biological species, economic species and digital species inherit all properties from species concept. The major differences between them are their living environment, commutative object and rule.

The instance of biological species is human. They live in the environment of natural ecosystem and interact with other humans. As a member of the society, they must follow the common rules in the human society (such as law). (Fig. 3)

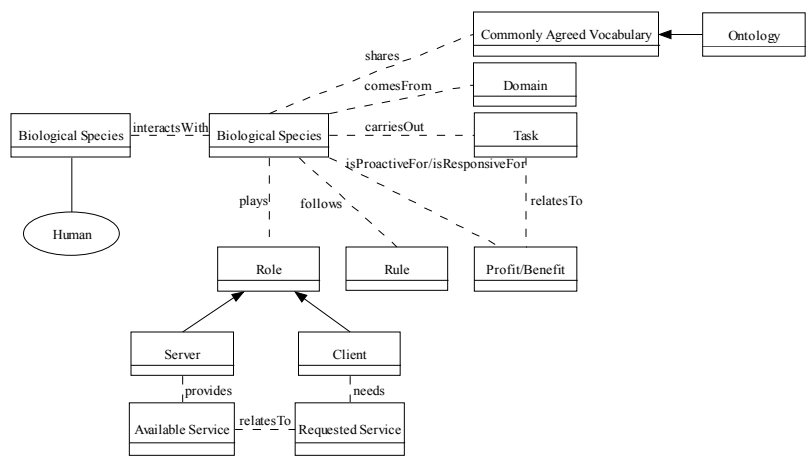

Figure 3. Biological Species Ontology

Organization is one example of Economic Species. Organizations exist in the business environment and their commutative scope is limited in themselves. Organization is the aggregation of individuals. Since organizations belong to certain industries, they must follow some domain-specific rules (such as industrial agreements). (Fig. 4)

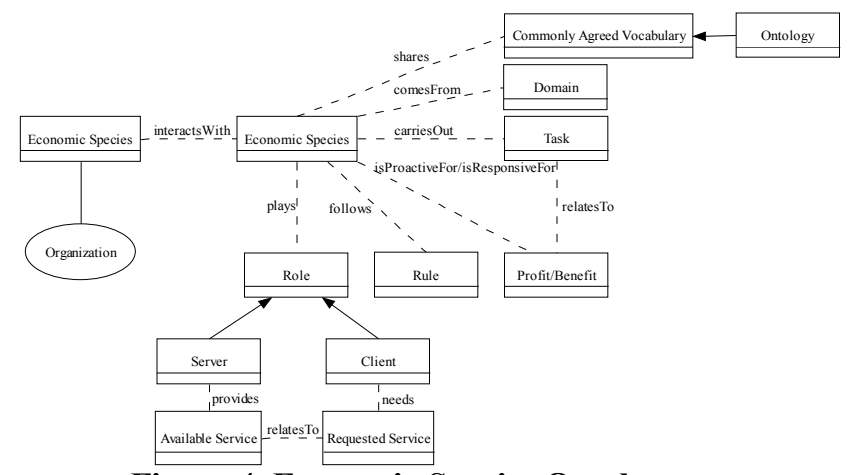

Figure 4. Economic Species Ontology

Digital Species mainly includes hardware and software. Digital species are connected by networks and they also communicate with each other via networks, thus their living environment is digital network. The rules exist in the network world are, e.g. protocols. (Fig. 5)

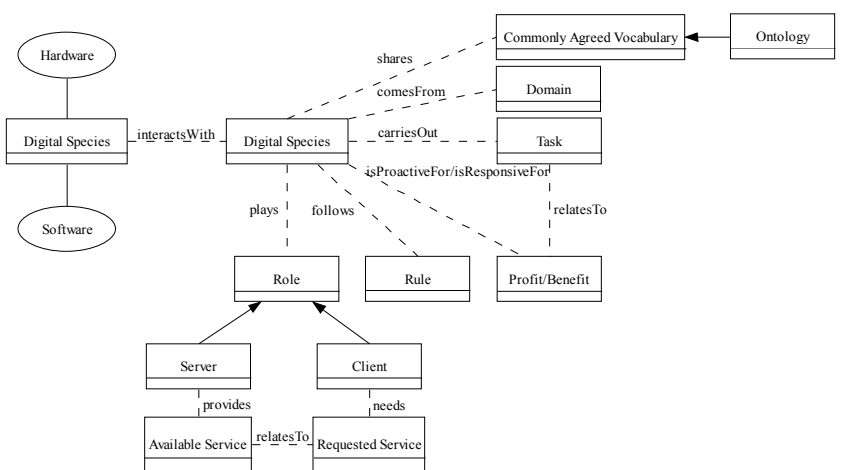

Figure 5. Digital Species Ontology

\subsection{Environment Ontology}

In an ecosystem, the environment supports all basic requirements for species' survival and evolution. However, species do not need to take care of their living environment. Differed from an ecosystem, there is bidirectional relationship between environment and species in digital ecosystem. Apart from the environment as the foundation for species' living which supports services to species, species also need to take care of the environment by means of providing technologies [1].

In the environment of digital ecosystem, the environment can be defined as the conceptualization of Environment that is supported by Technologies and has the specifications of Open, Loosely Coupled, Demand-driven, Domain-Clustered, Self-organizing, and Agent-based. The Environment Ontology is shown in Fig. 6 through the use of notation system. 


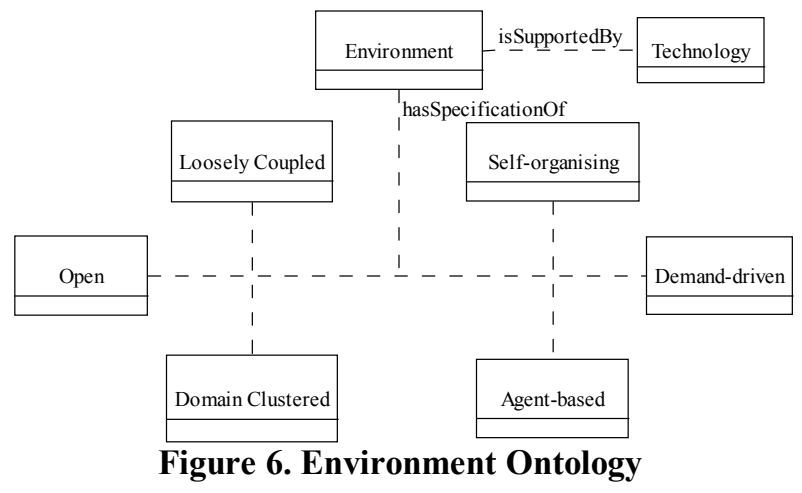

We present the Environment Ontology as the combination of ontology name and a tuple where the elements of the tuple can be complex elements as defined below:

Environment [Environment, Technology, Open, Loosely Coupled, Demand-driven, Domain Clustered, Self-organizing and Agent-based] where

'Environment' refers to definition above.

'Technology' will be defined in the next section.

'Open' refers to a transparent environment where all interactions are visible [4].

'Loosely Coupled' refers to a freely bound and open relationship between species in digital ecosystem [4].

'Demand-driven' means that species actively join in a community on their own interest [4].

'Domain Clustered' refers to an environment which consists of the field where some species have common interest [4].

'Self-organizing' means that species are capable of acting autonomously, making decisions and carrying out tasks in digital ecosystem [4].

'Agent-based' refers to an environment which contains human individuals, information technologies and tools that facilitate interaction and knowledge sharing along with the resources that help maintain synergy among human beings and organizations [4].

\subsection{Technology Ontology}

The underlying technologies for digital ecosystem are facilitated by the three types of species and these technologies provide services to digital ecosystem. Technologies have three main categories - extended web architecture, ontology-based knowledge sharing and a swarm intelligence-based self-organizing recommendation system. The concept of extended web architecture will be discussed in next section. Ontology-based knowledge sharing is to use ontological concepts as the major means to share multidisciplinary knowledge between different species. Swarm intelligence-based recommendation system is a platform on which species utilize swarm intelligence to cooperate and communicate with each other [4]. The ontology of swarm is defined in the following section.

The graph of Technology Ontology is shown in Fig. 7 through the use of notation system.

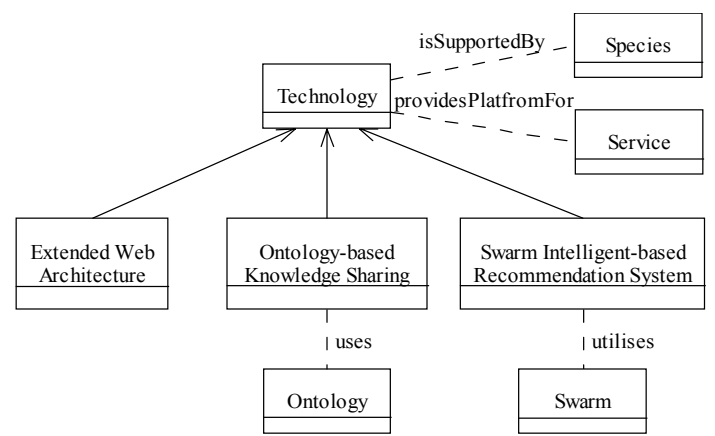

Figure 7. Technology Ontology

\subsection{Extended Web Architecture Ontology and its subcomponent ontologies}

Extend web architecture of digital ecosystem contains five basic subclass architectures which are client server architecture, peer-to-peer architecture, grid architecture, mobile and ad-hoc architecture and web-service architecture. Digital ecosystem architecture is constructed by the optional one or the optional combination of the architectures, which is a flexible and self-organizing network environment. The detailed infrastructure of digital ecosystem architecture refers to Soluta.net (www.digital-ecosystem.org). What we study in this paper is the conceptual model of digital ecosystem architecture which is shown in Fig. 8 .

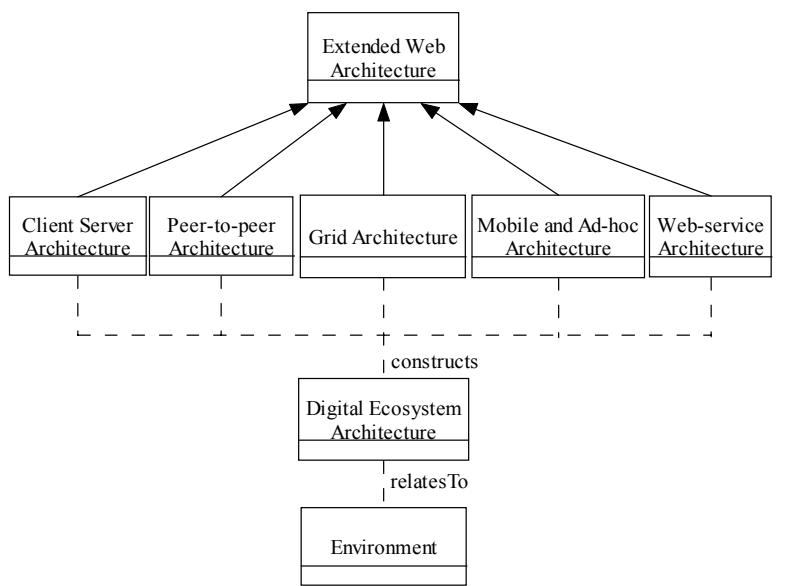

Figure 8. Extended Web Architecture Ontology

In this section, we will define the ontological models for the five basic subcomponents of extended web architecture in digital ecosystem. 
In client-server architecture, a server is centered and fully controls the communication between surrounded clients; on the other hand, the function of each client also depends on the function of server. The role of client and server is fixed and is not able to be changed [4]. (Fig. 9)

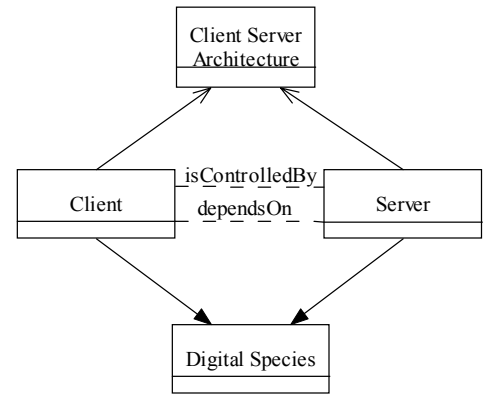

Figure 9. Client Server Architecture Ontology

Peer-to-peer network consists of distributed peers which has same role and functions. There is no centralized server in this network. The communicating ability and computing powers of peer-to-peer network relies on the peers in the end of each connection [6]. (Fig. 10)

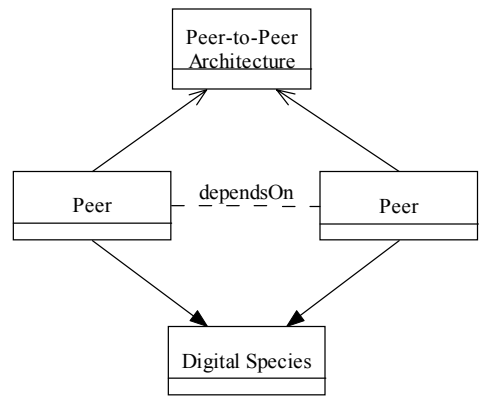

Figure 10. Peer-to-Peer Architecture Ontology

Grid architecture aggregates all geographically dispersed and autonomous resources and runs dynamically based on the capability of each node in a grid [7]. The difference between P2P and grid is, to the former, there is a centralized resource manager who manages all peers which work cooperatively; to the latter, there are distributed resource managers for each node, which supports the integration of heterogeneous systems [4]. (Fig. 11)

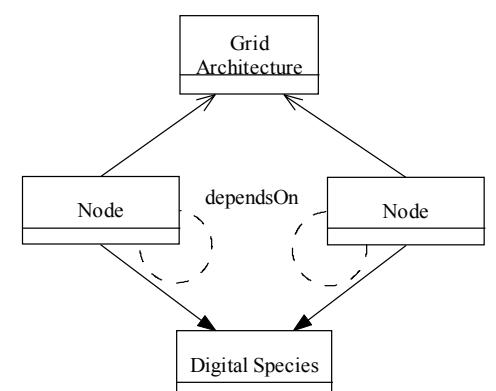

Figure 11. Grid Architecture Ontology

Mobile and ad-hoc network has the similar specifications - their connection is flexible and the main components of their network include compact devices and a server. The centralized server is fixed and controls other devices in the period of connection. In mobile network, the geographical location of compact devices is flexible. In ad-hoc network, the connection between compact devices and server is temporary. The compact devices' communication and other functions depend on the server's function. (Fig. 12)

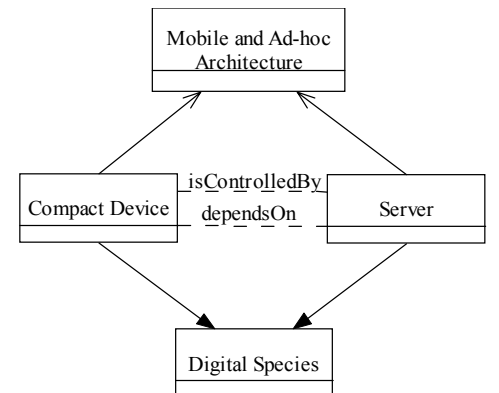
Figure 12. Mobile and Ad-hoc Architecture
Ontology

Web-service architecture involves three basic components - service requester, service broker and service provider. A service provider is to publish an available service to a service broker. A service requester is to request a service from a service broker. The mission of a service broker is to find and bind a proper service requester for a service provider, or a proper service provider for a service requester [8]. Meanwhile, service broker is centralized, and service requesters and service providers are distributed [4]. (Fig. 13) 


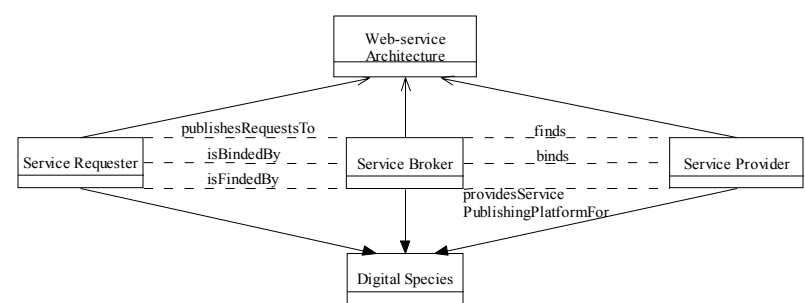

Figure 13. Web-service Architecture Ontology

\subsection{Swarm Ontology and Leading Species Ontology}

In digital ecosystem, as we discussed in the prior section, due to the specification of self-organizing and collaboration, species may form an organization with flexible structures to cooperate and communicate. Here a concept - swarm is defined by Chang and West, which is 'a set of species which has common characteristic and is able to interact and engage directly or indirectly with each other' [4]. With the swarm intelligence technology, species that have common interest may group and cooperate, to share a problem and to collaboratively carry out a task. The structure of swarm may vary from time to time, which depends on its mission. For example, a hierarchical swarm is convenient for organizational management and the definition of communication channels; a linear swarm is beneficial for species to efficiently execute sequential tasks or flow operations; a circular swarm is useful for species to cooperate and comprehensively tackle issues.

In a swarm, a leader is chosen from species, to direct and facilitate the warm, and to represent the swarm to interact with other swarms [4]. Besides these functions, the leading species has same features as other species.

The ontological representation of Swarm Ontology and Leading Species Ontology is shown in Fig. 14 and Fig. 15.

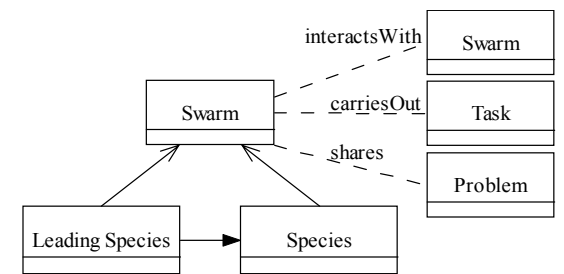

Figure 14. Swarm Ontology

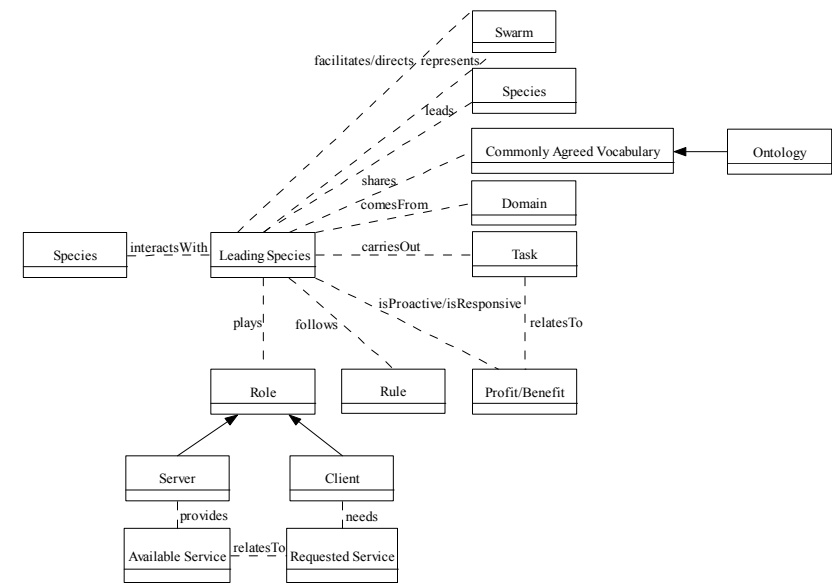

Figure 15. Leading Species Ontology

\section{Conclusion and future works}

In this paper, to solve the ambiguous problem in defining digital ecosystem, we use ontology to represent the conceptual model of digital ecosystem and its subcomponents, based on the theories presented by Chang and West. The outcome we achieve in this paper is concluded as below:

This paper proposes and explains the concept of digital ecosystem and its subclass concepts - Species and Environment. In addition, the specifications of Species - Natural Species, Economic Species and Digital Species are defined through use of a special notation system. Furthermore, we define the concept about Technology and its subcomponents that support the digital ecosystem environment, including the five categories of extended web architectures which construct the mainframe of digital ecosystem architecture, and the swarm intelligence as the basic organizational style in digital ecosystem.

The limitations in our research are drawn as below:

This paper chooses only one digital ecosystem theory (DEBII) as its research goal, which supports limited knowledge to researchers.

The ontological representation towards services provided by digital ecosystem is brief and surface, which needs more detailed and deeper research in the future.

Our conceptual model has not been realized and validated in the real world.

Thus, against the above drawbacks in our research, in the future, we will extend our ontology design to deeper sub-structure and combine the theory of Soluta.net to our prototype, in order to create a unified and widely recognized concept model. Moreover, we will research the function of digital ecosystem towards species by case study and literal research. Finally, we will prototype the ontological model in Protégé-owl 
and validate it in the real world by collecting other researchers' responses.

\section{References}

[1] E. Chang, T.S. Dillon and F.K. Hussain, Trust and Reputation for Service Oriented Environments-Technologies for Building Business Intelligence and Consumer Confidence, John Wiley \& Sons, Brisbane, 2005.

[2] E. Chang, M. Quaddus and R. Ramaseshan, "The Vision of DEBI Institute: Digital Ecosystems and Business Intelligence", DEBII, 2006.

[3] P. Ferronato, "Introduction to the Digital Business Ecosystem Project", Proceedings of MDA Technical Forum, Tokyo, 2004.
[4] E. Chang and M. West, "Digital Ecosystem - A Next Generation of the Collaborative Environment", Proceedings of iiWAS 2006, Yogyakarta, 2006.

[5] N. Guarino, "Ontology-driven Conceptual Modeling", Proceedings of 21st International Conference on Conceptual Modelling, Tampere, 2002, p.10.

[6] D.C. Verma, Legitimate Applications of Peer-to-Peer Networks, John Wiley \& Sons, Inc., 2004, pp. 1-17.

[7] "Grid Computing Info Centre", vol. 2007, R. Buyya, Ed.: GRID Infoware.

[8] "Web Services Architecture", vol. 2007, D. Booth, H. Haas, F. McCabe, E. Newcomer, M. Champion, C. Ferris, and D. Orchard, Eds.: W3C, 2004. 